\title{
Role of Simplex Lattice Statistical Design in the Formulation and Optimization of Microemulsions for Transdermal Delivery
}

\author{
Sureewan Duangjit,,$^{a \dagger}$ Leilah Maria Mehr, ${ }^{a, b}$ Mont Kumpugdee-Vollrath, ${ }^{b}$ and \\ Tanasait Ngawhirunpat*,a \\ ${ }^{a}$ Faculty of Pharmacy, Silpakorn University, Sanamchan Palace Campus; Nakhon Pathom 73000, Thailand: and \\ ${ }^{b}$ Faculty of Pharmaceutical and Chemical Engineering, University of Applied Sciences; Berlin, Germany. \\ Received July 30, 2014; accepted September 22, 2014; advance publication released online October 3, 2014
}

Microemulsions (ME) have gained attention as an alternative pharmaceutical formulation for transdermal delivery systems. However, the complicated relationships between various ME compositions (causal factors) and their characteristics (response variable) have not been fully comprehended. To overcome this problem, the design and development of ME for transdermal delivery was performed in our study using Design Expert $^{\circledR}$ Software. The model formulations of ME were prepared according to the ME region obtained from pseudo-ternary phase diagrams using the simplex lattice design as an optimization technique. In this study, ketoprofen-loaded ME composed of oleic acid, Cremophor ${ }^{\circledR}$ RH40, ethanol and water were prepared, and their characteristics (e.g., size, charge, conductivity, $\mathrm{pH}$, viscosity, drug content, loading capacity and skin permeation flux) were evaluated. The ME having an appropriate skin permeation flux was used as the basis for optimization. The skin permeation flux of the experimental ME was very close to the flux predicted by Design Expert ${ }^{\circledR}$ Software and was significantly greater than that for the commercial product. Possible mechanisms for the enhancement of the skin permeation of the ME were also investigated using Fourier transform infrared (FT-IR) spectroscopy and X-ray diffraction (XRD). This finding provided an understanding of the relationship between the causal factors and response variables, as shown in the response surfaces. Moreover, these results indicated that the simple lattice design was beneficial for the pharmaceutical development of ME for transdermal delivery.

Key words simplex lattice design; microemulsion; optimization; transdermal drug delivery; Fourier transform infrared (FT-IR) spectroscopy; X-ray diffraction (XRD)

Colloidal drug delivery carriers such as liposomes, niosomes, micelles, microemulsions, nanospheres, liquid crystal and particulate dispersions consisting of small particles of 10 to $400 \mathrm{~nm}$ diameters have exhibited great promise as drug delivery systems. Colloidal drug delivery carriers are one of the most important entities in pharmaceutical formulations designed primarily for targeted drug delivery systems. Using colloidal drug carriers for targeted drug delivery could minimize systemic side effects and also improve the therapeutic efficacy of various drugs. ${ }^{1)}$

Self-emulsifying systems known as microemulsions (ME) are optically isotropic transparent colloidal systems consisting of two immiscible liquid systems stabilized by surface tension reducing agents (surfactants). ${ }^{2)} \mathrm{ME}$ consist of an oil phase, water phase and surfactant or a mixture of surfactants, usually in combination with a co-surfactant, that spontaneously emulsify when mixed in optimal ratios. ME have long been utilized as drug delivery systems in various routes of delivery including topical, ${ }^{3)}$ oral, ${ }^{4)}$ ocular, $^{5)}$ nasal ${ }^{6)}$ and parenteral ${ }^{7)}$ routes. Moreover, ME have gained considerable interest as an alternative pharmaceutical formulation for transdermal delivery because of several advantages. ${ }^{2)}$ First, the incorporation of surfactant and co-surfactant in ME may introduce a high solubilizing capacity to incorporate a large amount of drugs in the formulation. Second, the surfactant and co-surfactant in $\mathrm{ME}$ may act as penetration enhancers, resulting in the disruption of major skin barrier functions of the stratum corneum. Third,

The authors declare no conflict of interest

${ }^{\dagger}$ Present address: Faculty of Pharmaceutical Sciences, Ubon Ratchathani University; Ubon Ratchathani 34190, Thailand. the skin permeability of drug-loaded ME may be increased by modifying the affinity of the drug to the internal phase in the $\mathrm{ME}$, to enhance dissolving or partitioning into the stratum corneum. Moreover, ME are not only highly efficient for transdermal delivery but also highly stable, being thermodynamically stable ${ }^{8)}$ compared to other carrier systems.

Previously, the development of ME has been based on trial and error techniques to obtain an acceptable ME formulation with multiple desirable characteristics (e.g., high efficacy, high stability and high safety). The complicated relationships between various ME compositions (causal factors; $X_{n}$ ) and their characteristics (response variable; $Y_{n}$ ) were not fully comprehended. A high concentration of a mixture of surfactants may relate to high skin permeability efficacy; however, skin irritation should be concurrently considered. Therefore, an optimization technique based on a computer rationale was a powerful method for the development of ME with optimal characteristics. Moreover, the complicated relationship between the causal factors and ME characteristics could be easily understood by using the computer program.

This study investigates the development of ME for the transdermal delivery of ketoprofen (KP). In the development of $\mathrm{ME}$ for transdermal delivery, it is important to design an optimal ME formulation that has sufficient skin permeation (maximum efficacy) with minimum surfactant/co-surfactant system levels (minimum skin irritation). The challenge in achieving this objective is to discover the optimal ME formulation for the transdermal delivery of KP by using the computer program. The model ME formulations for the transdermal delivery of KP were obtained by using a simplex lattice statistical design based on the ME regions obtained from 
(A)

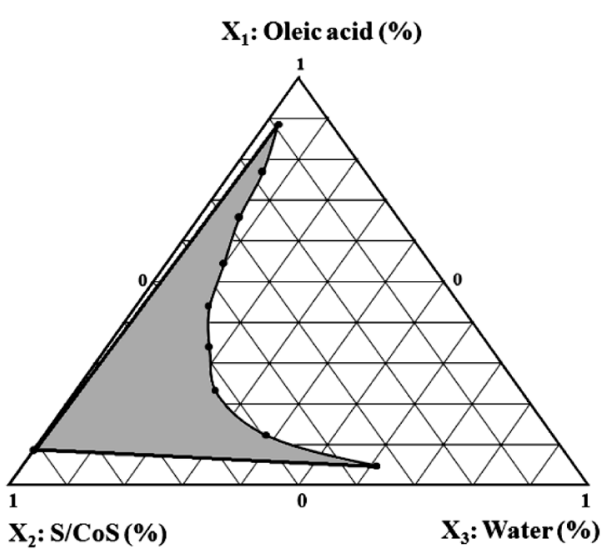

(B)

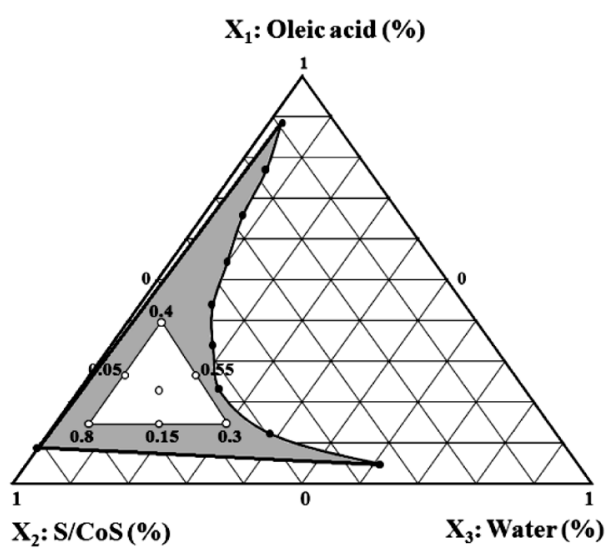

Fig. 1. The Phase Diagrams

(A) Pseudo-ternary phase diagram of microemulsions (ME). (B) Simplex lattice design for the three factors under the ME region. Eight formulations were prepared according to the results obtained using the simplex lattice design with duplicate centroids.

pseudo-ternary phase diagrams. The KP-loaded ME composed of oleic acid, Cremophor ${ }^{\circledR}$ RH40, ethanol and water were prepared. Their characteristics, e.g., size, charge, conductivity, $\mathrm{pH}$, viscosity, drug content, loading capacity and skin permeation flux were investigated. The ME were optimized using Design-Expert ${ }^{\circledR}$ Software and then prepared and compared with the commercially available product. Finally, possible mechanisms for the enhancement of skin permeation of the ME was evaluated and confirmed by Fourier transform infrared (FT-IR) spectroscopy and X-ray diffraction (XRD).

\section{MATERIALS AND METHODS}

Materials Oleic acid, KP and indomethacin was supplied by Sigma-Aldrich Production GmbH, (Buchs, Switzerland). Polyethylene glycol (PEG)-hydrogenated castor oil (Cremophor $^{\circledR}$ RH 40) was obtained from NS group (Bangkok, Thailand). Absolute ethanol (99.9\%) was purchased from Qrec Chemical Co., Ltd. (Chonburi, Thailand). All other chemicals and solvents were of analytical reagent grade.

Construction of the Pseudo-Ternary Phase Diagram The pseudo-ternary phase diagram of three components (oil phase, water phase and emulsifier phase) were constructed using aqueous titration method. ${ }^{9)}$ The emulsifier phase composed of the surfactant (S) and co-surfactant $(\mathrm{CoS})$ mixture at weight ratios of $1: 1,1: 2,1: 3$ and $1: 4$ were dissolved in oil phase, in the ratio of $1: 9,2: 8,3: 7,4: 6,5: 5,6: 4,7: 3,8: 2$ and $9: 1$ in glass vials at room temperature. Each ratio of the $\mathrm{S} / \mathrm{CoS}$ mixture and oil phase was titrated continuously drop by drop with distilled water, using a burette and gentle magnetic stirring until the solution became turbid. The behavior of each ternary phase system during the titration was visually observed. The percentage of each component in the ternary phase system was calculated, and the phase diagrams were plotted on triangular co-ordinates to construct the pseudoternary phase diagrams. ${ }^{10}$ )

Experimental Design The simplex lattice design was used for the optimization of pharmaceutical products in accordance with previous studies. ${ }^{11,12)}$ Three components in the ME, including the oil phase $\left(X_{1}\right)$, emulsifier phase $\left(X_{2}\right)$ and water phase $\left(X_{3}\right)$, were selected as causal factors. Based on the ME region obtained from the pseudo-ternary phase diagram shown in Fig. 1A, the upper and lower limits of the levels of each component were set as follows:

$$
\begin{gathered}
15 \leq X_{1} \leq 40(\%) \\
55 \leq X_{2} \leq 80(\%) \\
5 \leq X_{1} \leq 30(\%) \\
X_{1}+X_{2}+X_{3}=100(\%)
\end{gathered}
$$

Therefore, the feasible experimental region in the simplex lattice design was the largest triangular shape under the $\mathrm{ME}$ region. The components in the ME system were studied by varying their concentration simultaneously, and the total concentration of the three components was adjusted to $100 \%$, as defined in Eqs. (1)-(4).

Microemulsion Preparation The ME were prepared according to the formulation obtained from the simplex lattice design under the ME regions in the pseudo-ternary phase diagrams. As shown in Fig. 1B, 8 model formulations (with duplicate of the centroid) of KP-loaded ME were prepared, composed of oleic acid as the oil phase, Cremophor ${ }^{\circledR} \mathrm{RH} 40$ as the surfactant, absolute ethanol as the co-surfactant and distilled water as the water phase (Table 1). Previous studies indicated that oleic acid and Cremophor ${ }^{\circledR}$ RH 40 improve the transdermal delivery of various drugs. ${ }^{9,13)}$ All components were accurately weighed and mixed thoroughly, and the resulting $\mathrm{ME}$ were stored in airtight containers at room temperature, prior to further evaluation.

Determination of Droplet Size, Charge, Conductivity and pH The droplet size and charge of the ME were measured by photon correlation spectroscopy (Zetasizer Nano series, Malvern Instrument, U.K.). The samples were analyzed using a $\mathrm{He}-\mathrm{Ne}$ laser at $633 \mathrm{~nm}$. Thousand microliters of the ME were filled in disposable zeta cells. All analyses were performed at $25^{\circ} \mathrm{C}$, at least three independent samples were analyzed, and the droplet size and charge were measured in triplicate. The electrical conductivity of the ME was determined using conductivity meter (S230 SevenCompact ${ }^{\mathrm{TM}}$, Mettler Toledo, Switzerland), with at least three independent samples. The $\mathrm{pH}$ was measured using $\mathrm{pH}$ meter (S220 SevenCompact $^{\mathrm{TM}}$, Mettler Toledo, Switzerland) at room temperature $\left(25^{\circ} \mathrm{C}\right)$. The samples were analyzed in triplicate. 
Table 1. Experimental Design and Model Formulations of Ketoprofen-Loaded Microemulsions

\begin{tabular}{|c|c|c|c|c|c|c|}
\hline Formulation & $X_{1}$ & Oleic acid (\%) & $X_{2}$ & $\mathrm{~S} / \operatorname{CoS}(\%)$ & $X_{3}$ & Water $(\%)$ \\
\hline 1 & 0 & 15.0 & 1 & 80.0 & 0 & 5.0 \\
\hline 2 & 0.5 & 27.5 & 0.5 & 67.5 & 0 & 5.0 \\
\hline 3 & 1 & 40.0 & 0 & 55.0 & 0 & 5.0 \\
\hline 4 & 0.5 & 27.5 & 0 & 55.0 & 0.5 & 17.5 \\
\hline 5 & 0 & 15.0 & 0 & 55.0 & 1 & 30.0 \\
\hline 6 & 0 & 15.0 & 0.5 & 76.5 & 0 & 17.5 \\
\hline 7 & 0.33 & 23.5 & 0.33 & 63.5 & 0.33 & 13.5 \\
\hline 8 & 0.33 & 23.5 & 0.33 & 63.5 & 0.33 & 13.5 \\
\hline
\end{tabular}

Determination of Viscosity The viscosity of the ME was measured using a Brookfield Rheometer (Brookfield Engineering Laboratories, U.S.A.) fitted with a CP-40 spindle. The Brookfield Rheocalc operating software was used to control the rheometer. A minimum sample volume of $2 \mathrm{~mL}$ of $\mathrm{ME}$ was transferred into the viscometer chamber and the temperature was controlled at $25^{\circ} \mathrm{C}$ by circulating water bath. The time required for the fluid to flow between two specific points was measured and calculated in triplicate.

Determination of Drug Content and Loading Capacity The drug content and loading capacity of KP in the ME formulations were determined by HPLC. The KP-loaded ME were extracted with methanol at a $1: 1 \mathrm{v} / \mathrm{v}$ ratio. The mixture of $\mathrm{ME} /$ methanol was centrifuged at $10000 \mathrm{rpm}$ at $25^{\circ} \mathrm{C}$ for $10 \mathrm{~min}$. The supernatant was filtered with the $0.45 \mu \mathrm{m}$ nylon syringe filter and analyzed by HPLC. The drug content and loading capacity of KP in the ME was calculated according to the calibration curve and/or the following equation:

$$
\text { loading capacity }(\%)=\left(\frac{D_{\mathrm{t}}}{M_{\mathrm{t}}}\right) \times 100
$$

where $D_{\mathrm{t}}$ is the total weight of $\mathrm{KP}$ in the ME formulation and $M_{\mathrm{t}}$ is the total weight of ME formulation.

In Vitro Skin Permeation Study The shed snake skin of Naja kaouthia was used as a model membrane for the in vitro skin permeation study. ${ }^{14)}$ The shed snake skin was a kind gift from the Queen Saovabha Memorial Institute, Thai Red Cross Society, Bangkok, Thailand. It was stored at $-10^{\circ} \mathrm{C}$ prior to use. After thawing, the shed snake skin was cut and then immediately placed on the diffusion cell. A Franz diffusion cell with an available diffusion area of $2.01 \mathrm{~cm}^{2}$ was employed. The donor chamber was filled with the model ME $(1 \mathrm{~g})$, and the receiver chamber was filled with $6.5 \mathrm{~mL}$ of phosphate buffer saline $\left(\mathrm{pH} 7.4,32^{\circ} \mathrm{C}\right)$ under sink condition. At appropriate times, an aliquot of the receiver solution was withdrawn, and the same volume of fresh buffer solution was replaced in the receiver side. The concentration of KP in the aliquot was analyzed using HPLC.

The skin sample after the skin permeation study was characterized using a FT-IR spectroscopy (Nicolet 4700 spectrophotometer; Thermo Fisher Scientific, Waltham, MA, U.S.A.) and an X-ray diffractometer (MiniFlex II, Rigaku Co., Tokyo, Japan) for investigating the possible mechanism underlying the effect of the ME on the skin permeability of KP. Following the skin permeation study, the skin sample was prepared according to the procedure in the previous study. ${ }^{15,16)}$ Briefly, the skin sample was washed with distilled water, blotted dry and kept in desiccators. The spectrum of each skin sample was recorded in the range of $500-4000 \mathrm{~cm}^{-1}$ using a FT-IR spectrophotometer. Then, the same skin sample was cut into a small sheet, approximately $2 \mathrm{~cm} \times 2 \mathrm{~cm}$, and attached to an aluminum well sample holder. XRD was used with $\mathrm{Cu} K \alpha$, scanning from $2 \theta=5-45^{\circ}$. The voltage and current operated were $30 \mathrm{kV}$ and $15 \mathrm{~mA}$, respectively.

Determination of Ketoprofen Concentration The HPLC 1100 system (Agilent 1100 Series HPLC System, Agilent Technologies, U.S.A.) was used to analyze the samples. The analytical column was Eclipse XDB-C18 column (particle size $=5 \mu \mathrm{m}$; column dimension $=4.6 \times 150 \mathrm{~mm}$ ), and the mobile phase consisted of methanol and $0.1 \%$ phosphoric acid $(75: 25)$, set at a flow rate of $1 \mathrm{~mL} / \mathrm{min}$, and the UV detector was set at $254 \mathrm{~nm}$ for all determinations. The calibration curve for $\mathrm{KP}$ was in the range of $0.2-50 \mu \mathrm{g} / \mathrm{mL}$ with a correlation coefficient of 0.999 , using indomethacin as the internal standard. The intra-day and inter-day percent relative standard deviations ( $\%$ RSD) were less than $0.2 \%$.

Simultaneous Optimization Simplex lattice statistical design was used to estimate the relationship between the causal factors and response variables and statistically determine the optimal ME formulation. The experimental design was studied based on a three-component system: oil phase $\left(X_{1}\right)$, emulsifier phase $\left(X_{2}\right)$ and water phase $\left(X_{3}\right)$. The ME's characteristics, e.g., droplet size $\left(Y_{1}\right)$, charge $\left(Y_{2}\right)$, conductivity $\left(Y_{3}\right), \mathrm{pH}\left(Y_{4}\right)$, viscosity $\left(Y_{5}\right)$, drug content $\left(Y_{6}\right)$, loading capacity $\left(Y_{7}\right)$ and skin permeation flux $\left(Y_{8}\right)$ were taken as the response variables. The response surfaces of all model formulations were estimated and sketched by Design-Expert ${ }^{\circledR}$ Software, Version 8, Approved No. 009503 (Stat-Ease, Inc., MN, U.S.A.). The best fitting mathematics (e.g., linear, quadratic, cubic and special cubic) was suggested based on the model summary statistics: the standard deviation (S.D.), the multiple correlation coefficient $\left(R^{2}\right)$, the adjusted multiple correlation coefficient (adjusted $R^{2}$ ), the predicted multiple correlation coefficient (predicted $R^{2}$ ) and the predicted residual sum of square (PRESS), verified by Design-Expert ${ }^{\circledR}$ Software. The simultaneous optimal ME solution was predicted to have a droplet size as small as possible (nano-sized), non-charge (or a bit charge), conductivity (in range), $\mathrm{pH}$ (close to 5.5), viscosity (in range), drug content (maximum), loading capacity (maximum), skin permeation flux (maximum) and surfactant/cosurfactant system level (minimum). The predicted values and experimental values were evaluated to validate the reliability of the response surface.

\section{RESULTS AND DISCUSSION}

The ternary phase diagrams were constructed by varying the ratio of the $\mathrm{S} / \mathrm{CoS}$ mixture as $1: 1,1: 2,1: 3$ and $1: 4$. 
(A)

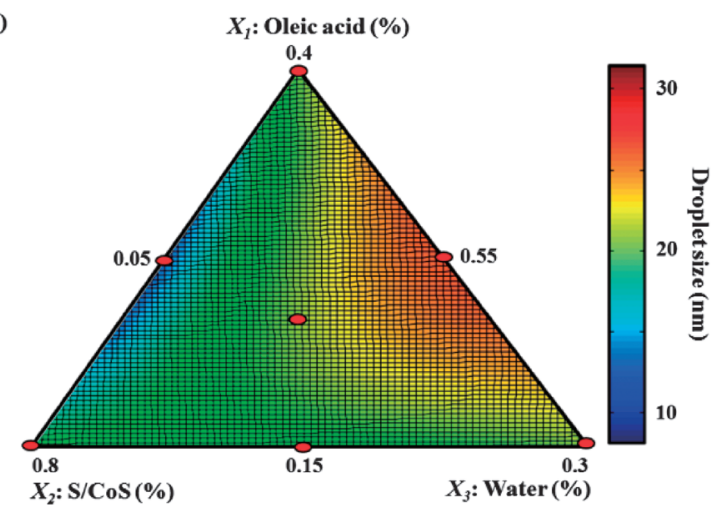

(C)

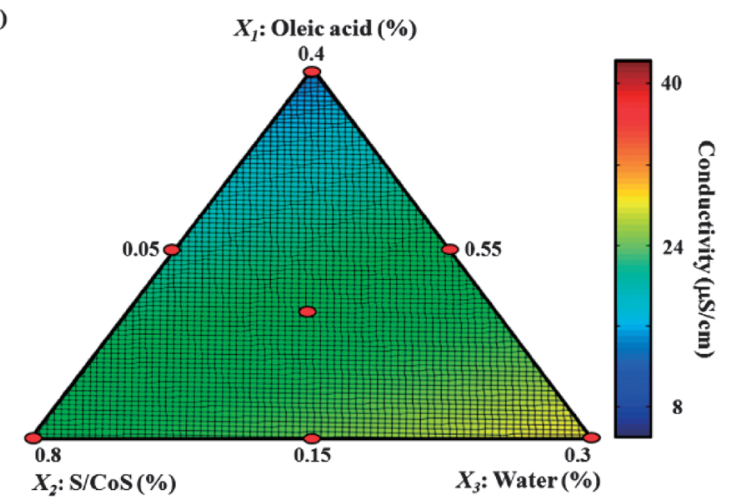

(E)

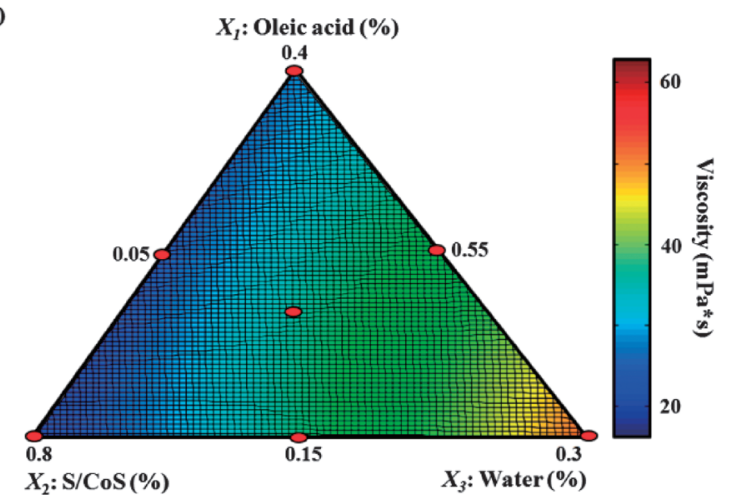

(G)

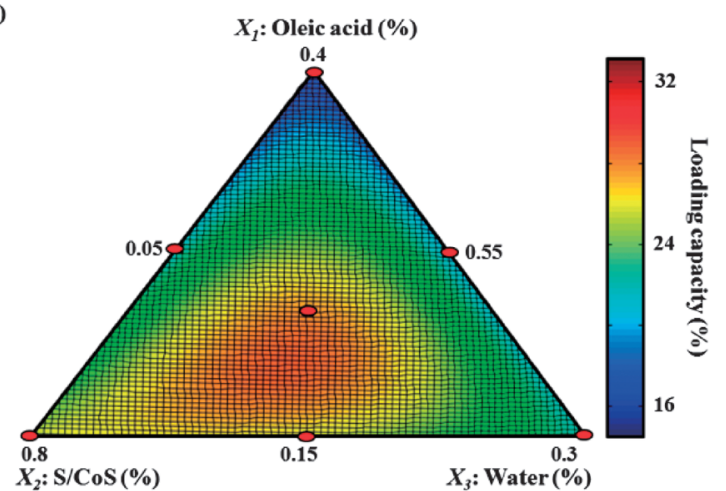

(B)

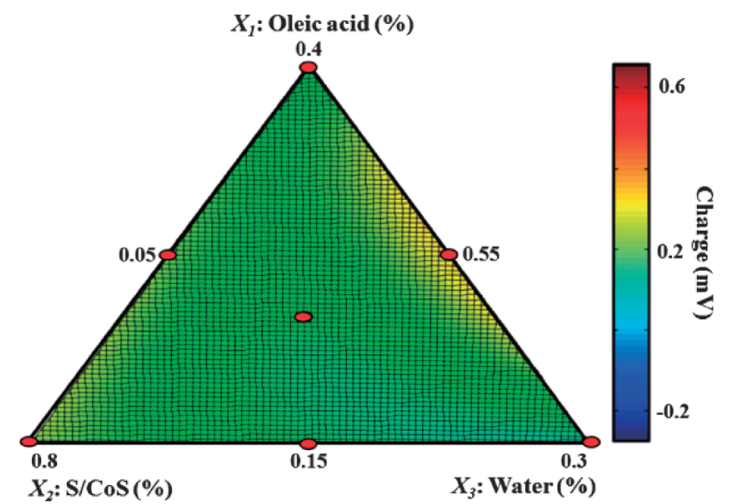

(D)

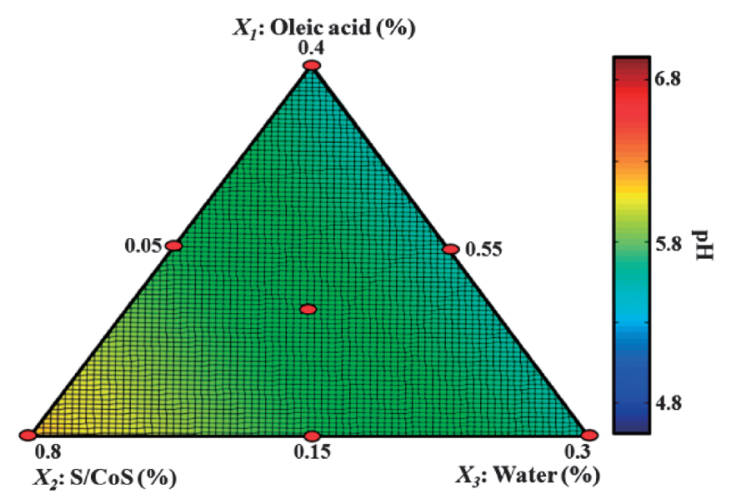

(F)

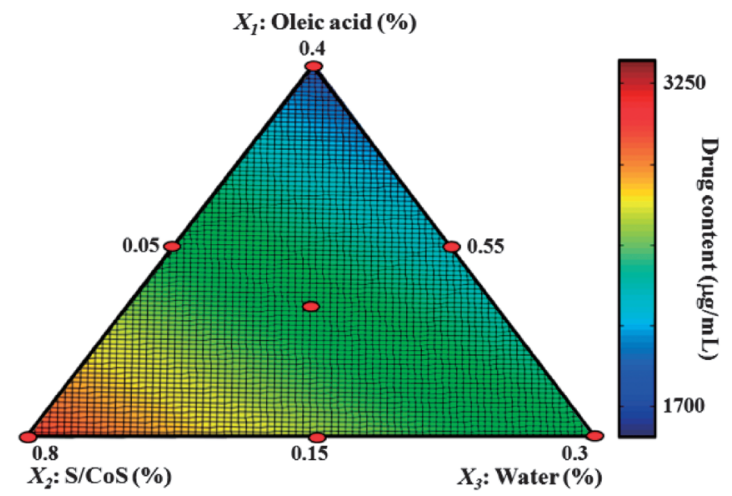

(H)

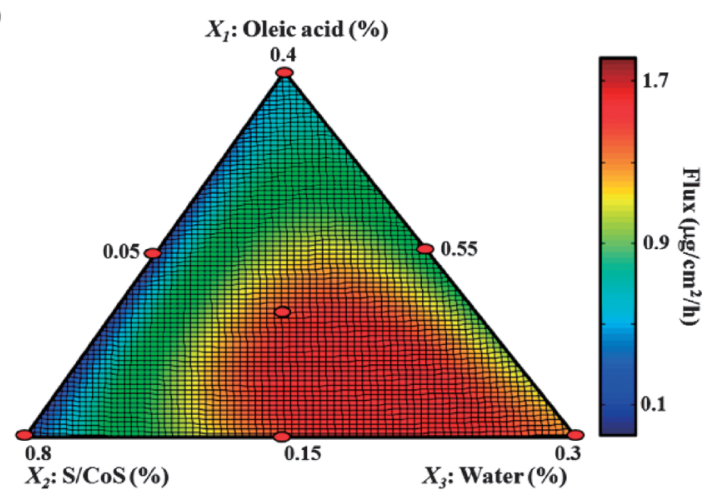

Fig. 2. The Response Surfaces of the ME's Characteristics Estimated by Design-Expert ${ }^{\circledR}$ Software, as a Function of the Percentage of Oleic Acid, $\mathrm{S} / \mathrm{CoS}$ and Water Included

(A) Droplet size, (B) charge, (C) conductivity, (D) pH, (E) viscosity, (F) drug content, (G) loading capacity and (H) skin permeation flux. 
(A)

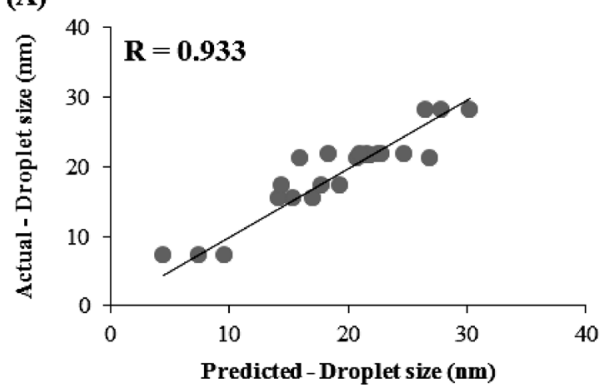

(C)

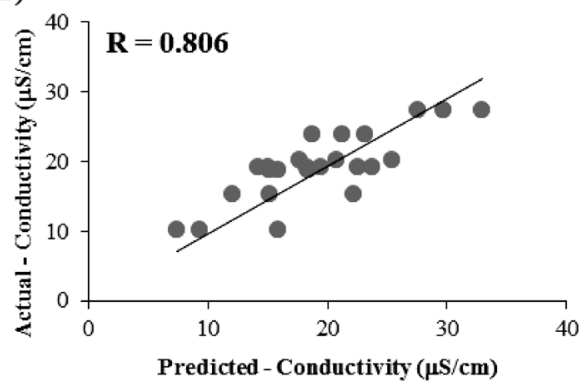

(E)

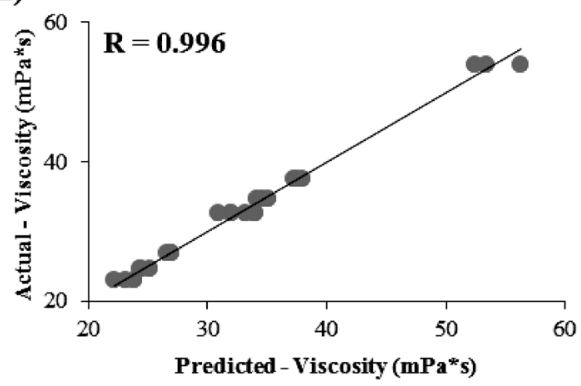

(G)

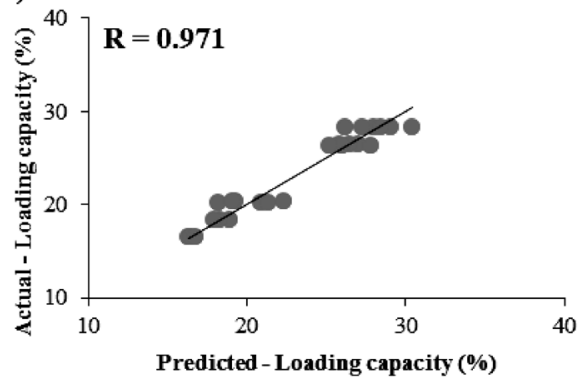

(B)

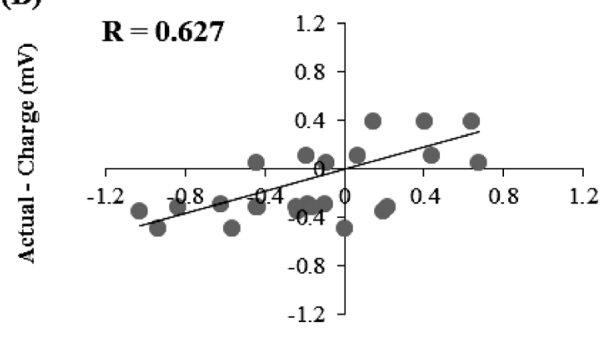

Predicted - Charge (mV)

(D)

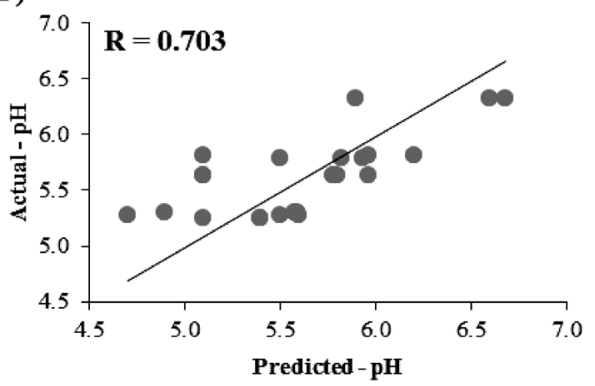

(F)

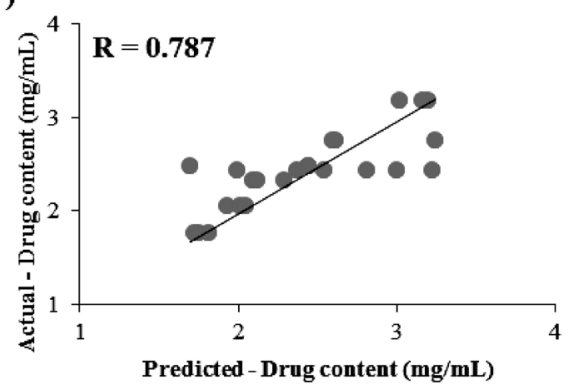

(H)

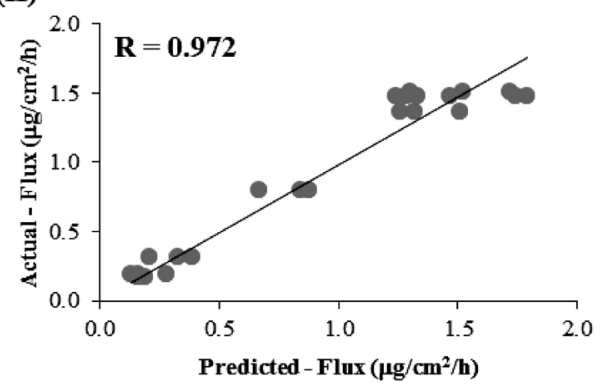

Fig. 3. The Linear Correlation Plot between the Predicted and Actual Values for Various Responses

(A) Droplet size, (B) charge, (C) conductivity, (D) pH, (E) viscosity, (F) drug content, (G) loading capacity and (H) skin permeation flux.

Based on the visual observation, the $1: 1 \mathrm{~S} / \mathrm{CoS}$ mixture was selected as the largest ME region. The shaded area of the ternary phase diagram indicates the ME region, while the non-shaded area shows the turbid region, as shown in Fig. 1A.

Prediction of Response Surface Using Design Expert ${ }^{\circledR}$ The response surfaces for each response variable were predicted by Design Expert ${ }^{\circledR}$, based on the experimental data. The appropriate concentration of oleic acid (oil phase), Cremophor ${ }^{\circledR}$ RH40: ethanol (S/CoS) (emulsifier phase) and water (water phase) were varied according to the ME region obtained from the pseudo-ternary phase diagram using the sim- plex lattice design, as shown in Fig. 1B. Eight model formulations of ME were prepared. The concentration of oleic acid, $\mathrm{S} / \mathrm{CoS}$ and water were selected as causal factors. The characteristics of ME e.g., droplet size, charge, conductivity, $\mathrm{pH}$, viscosity, drug content, loading capacity and skin permeation flux were selected as the response variables. The response surface estimated by Design Expert ${ }^{\circledR}$ shows an obvious relationship between the causal factors and the response variables. ${ }^{17)}$

The effect of three ME components (oil, S/CoS, water) on the characteristics of KP-loaded ME is shown in Fig. 2. The response surface indicated that a decrease in $\mathrm{S} / \mathrm{CoS}$ resulted 
(A)

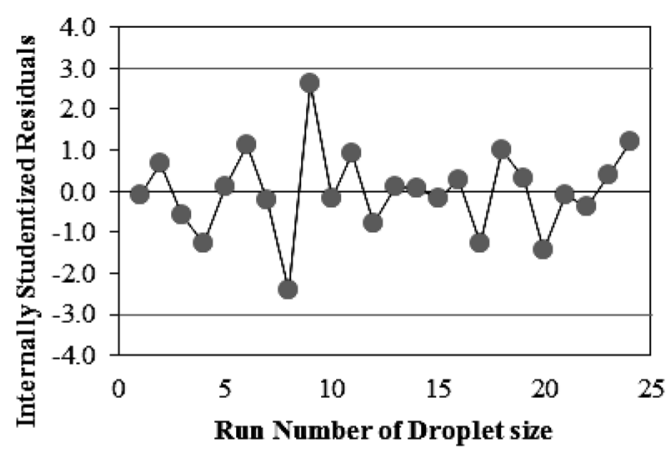

(C)

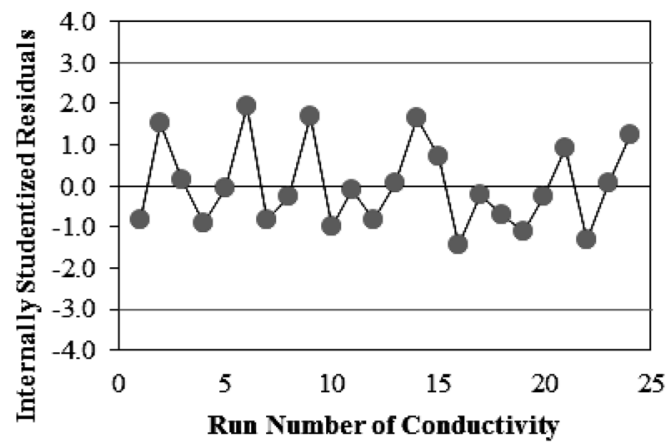

(E)

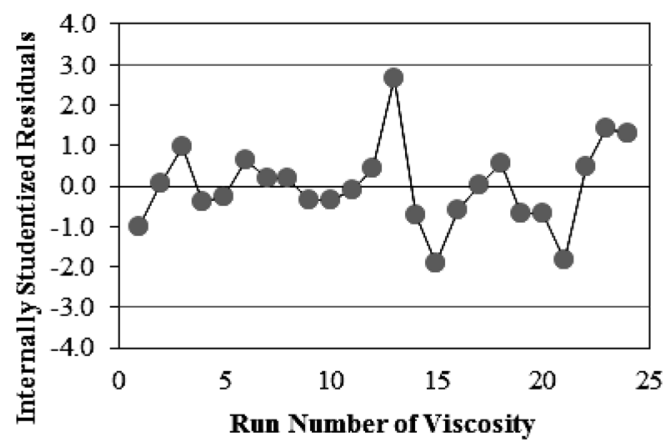

(G)

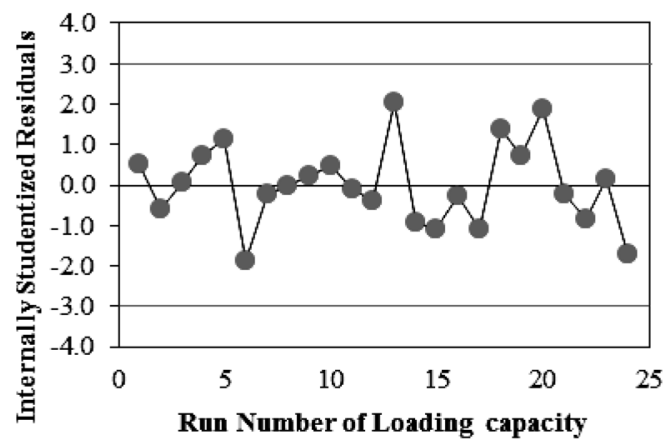

(B)

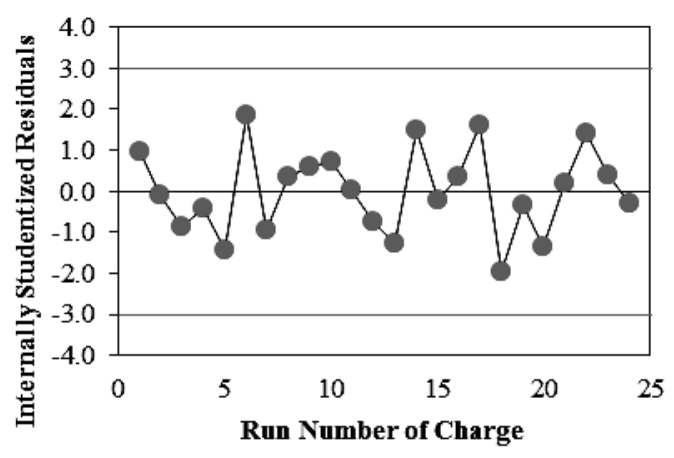

(D)

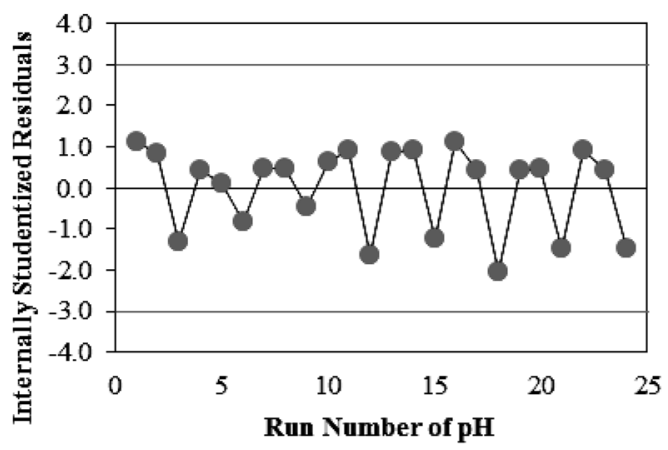

(F)

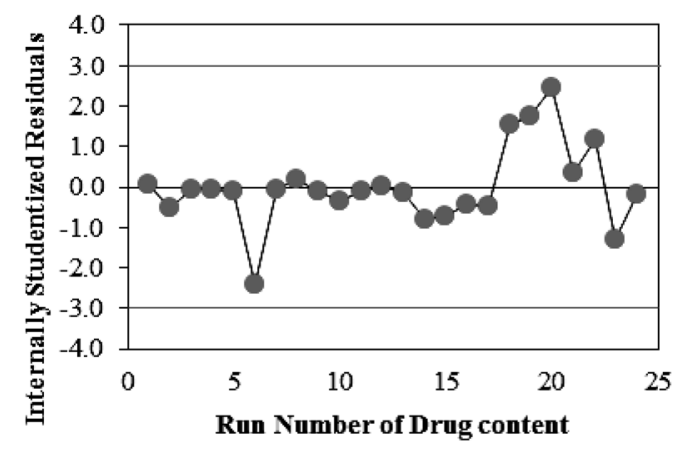

(H)

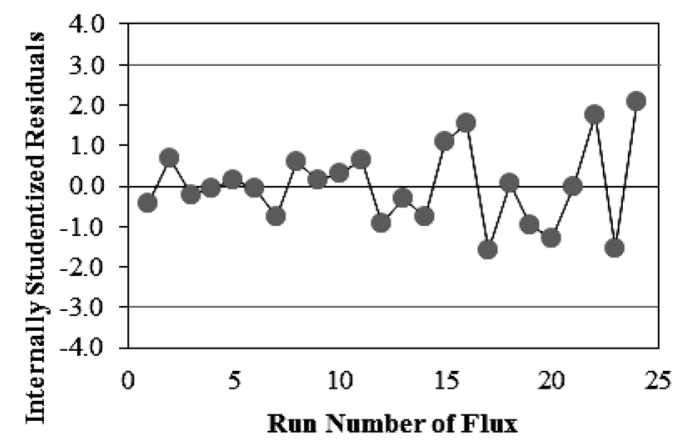

Fig. 4. The Corresponding Residual Plot between Run Number and Internally Studentized Residuals for Various Responses (A) Droplet size, (B) charge, (C) conductivity, (D) pH, (E) viscosity, (F) drug content, (G) loading capacity and (H) skin permeation flux. 
in a significant increase in droplet size, whereas an increase in $\mathrm{S} / \mathrm{CoS}$ resulted in a significant increase in drug content and loading capacity. Moreover, an increase of water resulted in a significant increase in conductivity, viscosity and skin permeation flux. Although the ME compositions might affect the charge and $\mathrm{pH}$ of the $\mathrm{ME}$ formulation, this could not be significantly observed in this study. This might be because the ME components used in the ME formulation have the same intrinsic physicochemical properties. The charge of water and ethanol is zero, or weakly negative (less than -1) or weakly positive (less than +1 ) in the experimental condition, while oleic acid and Cremophor ${ }^{\circledR}$ RH40 are non-ionic substances. Therefore, the difference in the ratio of each component might not significantly affect its total net charge. Moreover, the individual $\mathrm{pH}$ of each $\mathrm{ME}$ component used was in the same range ( $\mathrm{pH} 5-8$ ); thus, varying the ratios of the ME components might not significantly affect its net $\mathrm{pH}$.

The response surface of droplet size could be useful for preparing the ME with various droplet sizes. To prepare the $\mathrm{ME}$ with small droplet size, the percentage of water in the ME should be low; in contrast, a low level of $\mathrm{S} / \mathrm{CoS}$ resulted in ME with large droplet size. The response surface of conductivity was useful for classifying the types of ME: water-in-oil (W/O) and oil-in-water $(\mathrm{O} / \mathrm{W})$. The high level of oleic acid might result in the low conductivity of ME. ME formulations with low conductivity $(<10 \mu \mathrm{S} / \mathrm{cm})$ was classified as W/O ME, whereas ME with conductivity higher than $10 \mu \mathrm{S} / \mathrm{cm}$ was recognized as $\mathrm{O} / \mathrm{W} \mathrm{ME}{ }^{18)}$ This response surface could be used for predicting the types of all ME formulations in this region without the preparation of experimental ME. Moreover, the response surface of viscosity suggested that a high level of water significantly increases the viscosity of ME. The contact time of pharmaceutical formulations is directly affected by their viscosity, which could increase the therapeutic effect of the drug. ${ }^{19-21)}$ The drug content and loading capacity of the $\mathrm{ME}$ formulation could increase by increasing the level of $\mathrm{S} /$ $\mathrm{CoS}$ from medium to high. Because Cremophor ${ }^{\circledR}$ RH40 and ethanol act as solubilizing agents, increasing $\mathrm{S} / \mathrm{CoS}$ in the $\mathrm{ME}$ resulted in an increase in the amount of drug incorporated in the ME. The solubility of the drug in the ME depended on the solubilization capacity of the total ME system and on the ME structure. The structure of ME containing high surfactant levels (20-80\%) enables to incorporate large amounts of both lipophilic and hydrophilic drugs in the ME formulation. ${ }^{8}$ The response surface of skin permeation flux indicated that a low level of oil, medium level of $\mathrm{S} / \mathrm{CoS}$ and medium to high level of water ratio resulted in a significant increase in the flux of the ME formulations. Various studies suggested that an increase in some penetration enhancers in ME increased the skin permeability, ${ }^{22,23)}$ but not always. ${ }^{18,24)}$ This is because skin permeability appears to depend on other factors, e.g., specific formulation designs or drug concentration in the formulation (drug content). ${ }^{18)}$ The partition coefficient of a drug between the oil and water phase of ME at the high level of water region may appropriate for drug permeability. The solubility of KP in oleic acid and S/CoS was higher than in water. The high level of water in the ME may not prevent KP diffusion to the skin surface, ${ }^{25,26)}$ but KP in other ME regions at high levels of oleic acid or $\mathrm{S} / \mathrm{CoS}$ might prefer to localize in the ME, instead of permeating into the skin. ${ }^{27)}$ Furthermore, water is natural and safe and thus most widely used as a pen- etration enhancer to improve transdermal drug delivery, by increasing the water content of the tissue. ${ }^{28)}$ Generally, increasing skin hydration tends to increase the transdermal delivery of both hydrophilic and lipophilic drugs. High water levels in $\mathrm{ME}$ is not only to result in high efficacy but also to improve the safety of the ME formulation, simultaneously.

The linear correlation plot between the predicted and actual values for various response variables are shown in Fig. 3. The linear correlation coefficient is a statistical parameter used to define the linear relationship between the predicted and actual value, indicating the reliability and stability of the response surface. The linear correlation coefficient results for droplet size, viscosity, drug content, loading capacity and skin permeation flux were relatively high $(0.8-0.9)$, whereas some of them (charge and $\mathrm{pH}$ ) were quite low (0.6-0.7). The reliability of these results was confirmed by the corresponding residual plot between run number and internally studentized residuals for various response variables, as shown in Fig. 4. Based on the completely randomized run, the vertical spread of the internally studentized residuals was not out of the line from bottom-to-top, indicating that all points fall within the limits (at 95\% confidence level). Our results indicated that the Design Expert ${ }^{\circledR}$ successfully estimated the response surface that exhibited the relationship between the ME composition and the ME characteristics.

Optimization of ME The ME formulation of KP was optimized based on the experimental data using Design Expert ${ }^{\circledR}$ Software. The response variables criteria for estimating the optimal ME were set to determine the ME formulation with good efficacy for transdermal drug delivery. The constraint of optimization was formulated to maximize skin permeation flux (maximize efficacy) and to minimize the concentration of $\mathrm{S} / \mathrm{CoS}$ (minimize irritation) with equal importance and equal constraints in the triangular experimental region defined in Eqs. (1)-(4).

$X_{1}=19.22(\%), X_{2}=61.70(\%)$ and $X_{3}=19.08(\%)$ were estimated as the optimal ME formulation. Figure 5 shows the three dimensional (3D) response surface plot of the desirability of the optimal ME, which is located near the centroid of the simplex lattice design. The desirability of the prediction was 0.8184 . To evaluate the accuracy of the optimal ME formulation predicted by Design Expert ${ }^{\circledR}$, the ME formulation composed of the optimal composition ratio was prepared and

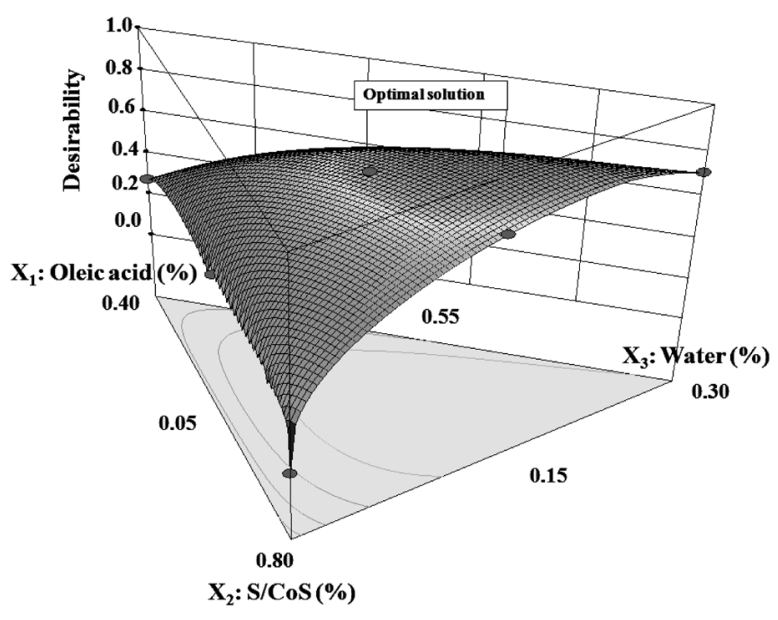

Fig. 5. The Three Dimensional (3D) Response Surface Plot of Desirability at the Prediction 
Table 2. The Response Variables of the Optimal ME

\begin{tabular}{|c|c|c|c|}
\hline Response & Predicted optimal ME & Experimental optimal ME & Bias $(\%)$ \\
\hline$Y_{1}(\mathrm{~nm})$ & 23.23 & $34.12 \pm 3.39$ & -31.92 \\
\hline$Y_{2}(\mathrm{mV})$ & -0.37 & $-0.34 \pm 0.05$ & 8.82 \\
\hline$Y_{3}(\mu \mathrm{S} / \mathrm{cm})$ & 22.56 & $22.10 \pm 1.49$ & 2.08 \\
\hline$Y_{4}$ & 5.51 & $5.52 \pm 0.01$ & -0.18 \\
\hline$Y_{5}(\mathrm{mPa} \cdot \mathrm{s})$ & 38.68 & $39.51 \pm 0.24$ & -2.10 \\
\hline$Y_{6}(\mu \mathrm{g} / \mathrm{mL})$ & 2462.81 & $2479.50 \pm 58.05$ & -0.67 \\
\hline$Y_{7}(\%)$ & 27.28 & $27.14 \pm 0.74$ & 0.52 \\
\hline$Y_{8}\left(\mu \mathrm{g} / \mathrm{cm}^{2} / \mathrm{h}\right)$ & 1.70 & $1.68 \pm 0.10$ & 1.19 \\
\hline
\end{tabular}

* Bias was calculated as $\{$ (predicted value-experimental value $) /$ experimental value $\} \times 100 \%$.

investigated experimentally. The ideal characteristics estimated by Design Expert ${ }^{\circledR}$ and the experimental characteristics measured for the optimal ME are shown in Table 2. The results indicated that the characteristics of the optimal ME demonstrated by the experiment were very close to the predicted values estimated by the computer program. The reliability of this study was displayed as the percentage bias. ${ }^{29)}$ Moreover, this result suggested that at the minimum $\mathrm{S} / \mathrm{CoS}$ level, the skin permeation flux of optimal ME was sufficiently high for the transdermal delivery of KP. A previous study reported that the skin permeation flux of meloxicam-loaded menthosomes ${ }^{30}$ ) and ondansetron hydrogels ${ }^{31)}$ predicted by computer program matched with the experiment flux measured by in vitro skin permeation studies.

Comparative Study of Optimal ME and Commercially Available Product Figure 6 shows the cumulative skin permeation per area and the skin permeation flux of $2.5 \% \mathrm{KP}$ gel (commercial product) and 2.5\% KP-loaded ME (optimal ME). The cumulative skin permeation per area of both formulations increased linearly after $3 \mathrm{~h}$. The skin permeation flux of KP through the skin sample was determined as a slope of the linear portion of the plot. The result indicated that the skin permeation flux for the optimal ME was significantly higher than that for the gel $(p<0.05)$. The ME system may promote skin permeability by various mechanisms. According to the skin permeation profile, some ME droplets may interact with the stratum corneum; this localization effect may result in increased permeation of the drug across the skin due to the high local concentration at the skin surface or the site of action. The other possible mechanism is that the fusion of the ME droplet to the skin results in a potential penetration-enhancing effect. However, the drug in the continuous phase might also partition into the skin, and the drug will consequently be released from the droplet to the continuous phase, compensating for the permeated drug as the free drug mechanism. ${ }^{8,32,33)}$ However, the mechanism of our ME in delivering the drug through the skin is not fully understood, and should be confirmed by further study.

The effect of gel and ME formulations on the stratum corneum of the skin sample was investigated by FT-IR and XRD. Moreover, the possible mechanisms of action of the ME were confirmed by FT-IR spectra and XRD pattern, as shown in Fig. 7. In the FT-IR spectra region between 2800 to $3000 \mathrm{~cm}^{-1}$ the methylene stretching $\left(\mathrm{CH}_{2}\right)$ bands were remarked as the most intensive spectra. The downward shifts in $\mathrm{CH}_{2}$ symmetric stretching (ca. $2850 \mathrm{~cm}^{-1}$ ) and asymmetric stretching (ca. $2920 \mathrm{~cm}^{-1}$ ) frequencies reflected the changes in hydrocarbon chain fluidity and conformational order or alkyl chain

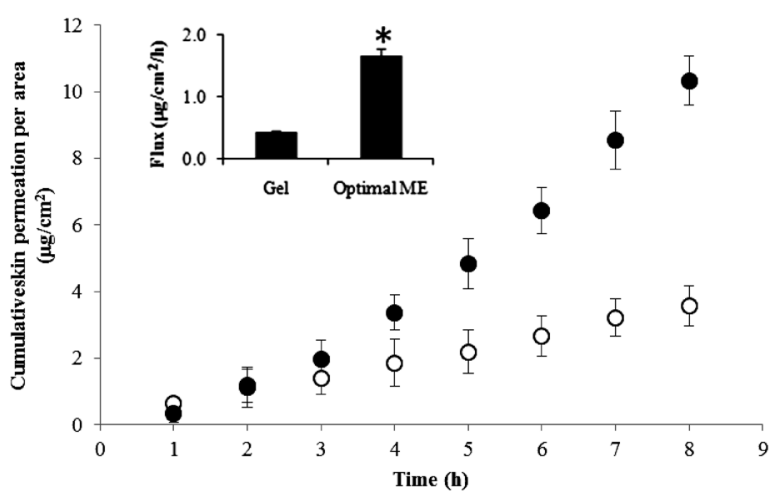

Fig. 6. The Skin Permeation Profile and Skin Permeation Flux of the Optimal ME and the Commercially Available Product

Each value represents the mean \pm S.D. $(n=3)$, * indicates statistical significance $(p<0.05)$. The white circle represents gel formulation and the fill circle represents optimal ME.

packing. ${ }^{34)}$ While the FT-IR spectra region between 1500 to $1700 \mathrm{~cm}^{-1}$ originate from amide I (mostly from $\mathrm{C}=\mathrm{O}$ stretch $(80 \%)$ ) and amide II (mostly mixed $\mathrm{N}-\mathrm{H}$ in plan bend $(60 \%)$ and $\mathrm{C}-\mathrm{N}$ stretch $(40 \%))$. The amide I band was split into doublet $\left(1620\right.$ to $\left.1690 \mathrm{~cm}^{-1}\right)$, but the amide II band was broad peak $\left(1510\right.$ to $\left.1560 \mathrm{~cm}^{-1}\right)$. The alteration in the shape of amide I and amide II was used as a marker for understanding the interaction and organization of hydrogen bonds in the polar interface. ${ }^{35)}$ In this study, the skin treated with gel formulations showed no difference in the FT-IR spectra, when compared to that from intact skin. In contrast, the $\mathrm{CH}_{2}$ symmetric stretching and asymmetric stretching peaks of the skin treated with the ME formulations were shifted from $2850 \mathrm{~cm}^{-1}$ and 2920 to $2849 \mathrm{~cm}^{-1}$ and $2918 \mathrm{~cm}^{-1}$, respectively. Additionally, the shape of the amide I and amide II bands was altered from 1620 to $1690 \mathrm{~cm}^{-1}$ to near regions 1640 to $1660 \mathrm{~cm}^{-1}$ and from 1510 to $1560 \mathrm{~cm}^{-1}$ to 1520 to $1550 \mathrm{~cm}^{-1}$, respectively, as shown in Fig. 7A. Furthermore, the X-ray diffractograms at $2 \theta=10$ and $20^{\circ}$ indicated the hexagonal packing of alkyl chains of lipid in the skin. ${ }^{36)}$ The XRD pattern of the skin treated with gel formulations and ME formulations was not significantly different from the intact skin as shown in Fig. 7B. However, when the X-ray diffractograms of all skin patterns were overlaid (as shown in the enlarge picture), the X-ray diffractograms showed that the intensity of the XRD pattern of the skin treated with the $\mathrm{ME}$ formulations was slightly decreasing and increasing at $2 \theta=10$ and $20^{\circ}$, respectively. These results suggested that $\mathrm{ME}$ formulations could deliver the drug through the skin, while the microstructure of the skin was not considerably disrupted. A previous study found that the effect of ethanol on the lipid 

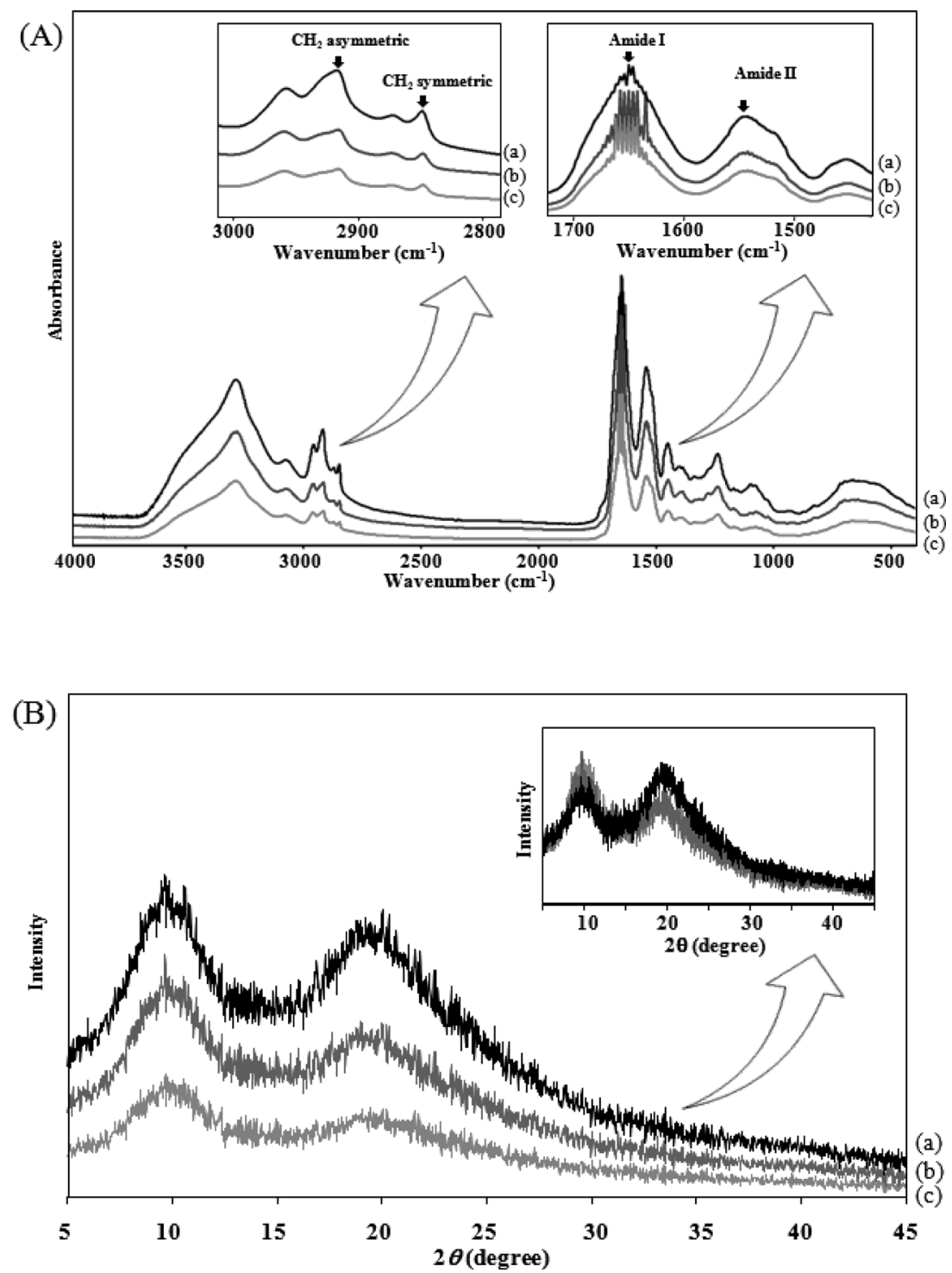

Fig. 7. Representative (A) FT-IR Spectra and (B) X-Ray Diffraction Patterns of (a) Intact Shed Snake Skin and (b) Shed Snake Skin Sample after Treatment with Gel Formulation (Commercial Product) and (c) ME Formulation

extraction in hairless mouse skin was obvious at $50 \%$ of ethanol or higher. ${ }^{37)}$ In the present study, the ethanol used was approximately $30 \%$; therefore, the extraction effect was not pronounced. These results indicated that the effect of ethanol in the ME was not the extraction of lipid (thus this was safe for skin) but the disruption of the organization of lipid structure for improving skin permeability; thus, the disruption of the organization of lipid structure may be affected by the other components of the ME.

\section{CONCLUSION}

Based on a simplex lattice statistical design, an optimal $\mathrm{ME}$ formulation for the transdermal delivery of KP was developed. The formulation was composed of oleic acid as the oil phase, Cremophor ${ }^{\circledR}$ RH40 as the surfactant, ethanol as the co-surfactant and water as the aqueous phase. Using Design Expert $^{\circledR}$ Software, the response surfaces indicated a clear relationship between the causal factors and the response variables. The optimal ME was defined as the formulation with an appropriate skin permeation flux and minimum $\mathrm{S} / \mathrm{CoS}$. This optimal formulation was estimated using a computer program, as well as determined experimentally. The experimental value of the skin permeation flux of the optimal ME was very close to the flux predicted by the computer program and was significantly greater than the commercial product. Considering the skin permeability of the optimal ME, we were successful in showing the feasibility of ME for the transdermal delivery of KP. Furthermore, the results indicated that the simple lattice design was beneficial as a powerful technique for the pharmaceutical development of ME for transdermal delivery.

Acknowledgments The authors gratefully acknowledge the Thailand Research Funds through the Basic Research Grant (Grant No. 5680016) and the Faculty of Pharmacy, Silpakorn University, Nakhon Pathom, Thailand for facilities and financial support. Finally, the authors would like to acknowledge Dr. Kampanart Huanbutta of the Faculty of Pharmaceutical Science, Burapha University, Thailand for computer analysis support. 


\section{REFERENCES}

1) Barratt GM. Therapeutic application $\mathrm{s}$ of colloidal drug carriers. Pharm. Sci. Technol. Today, 3, 163-171 (2000).

2) Fanun M. Microemulsions as delivery systems. Curr. Opin. Colloid Interface Sci., 17, 306-313 (2012).

3) Shalviri A, Sharma A, Patel D, Sayani A. Low-surfactant microemulsions for enhanced topical delivery of poorly soluble drugs. $J$. Pharm. Pharm. Sci., 14, 315-324 (2011).

4) Serdoz F, Voinovich D, Perissutti B, Grabnar I, Hasa D, Ballestrazzi R, Coni E, Pellegrini E. Development and pharmacokinetic evaluation of erythromycin lipidic formulations for oral administration in rainbow trout (Oncorhynchus mykiss). Eur. J. Pharm. Biopharm., 78, 401-407 (2011).

5) Kapoor Y, Chauhan A. Ophthalmic delivery of cyclosporine A from Brij-97 microemulsion and surfactant-laden $p$-HEMA hydrogels. Int. J. Pharm., 361, 222-229 (2008).

6) Piao H-M, Balakrishnan P, Cho H-J, Kimb H, Kim Y-S, Chung S-J, Shim C-K, Kim D-D. Preparation and evaluation of fexofenadine microemulsions for intranasal delivery. Int. J. Pharm., 395, 309-316 (2010).

7) Kale AA, Patravale VB. Development and evaluation of lorazepam microemulsions for parenteral delivery. AAPS PharmSciTech, 9, 966-971 (2008).

8) Pappinen S, Urtti A. Microemulsions in topical drug delivery. Percutaneous penetration enhancers. (Smith EW, Maibach HI eds.). CRC Press, New York, pp. 109-116 (2006).

9) Ngawhirunpat T, Worachun N, Opanasopit P, Rojanarata T, Panomsuk S. Cremophor RH40-PEG 400 microemulsions as transdermal drug delivery carrier for ketoprofen. Pharm. Dev. Technol., 18, 798-803 (2013).

10) Ahmad J, Mir SR, Kohli K, Amin S. Effect of oil and co-surfactant on the formation of Solutol HS 15 based colloidal drug carrier by Box-Behnken statistical design. Colloids Surf. A, 453, 68-77 (2014).

11) Patel DM, Patel NM, Pandya NN, Jogani PD. Gastroretentive drug delivery system of carbamazepine: Formulation optimization using simplex lattice design: A technical note. AAPS PharmSciTech, 8, E82-E86 (2007).

12) Prajapati S, Patel D, Patel C. Floating matrix tablets of domperidone formulation and optimization using simplex lattice design. Iran J. Pharm. Res., 33, 113-122 (2009).

13) Charoenputtakhun $P$, Opanasopit $P$, Rojanarata T, Ngawhirunpat $T$. All-trans ratinoic acid-loaded lipid nanoparticles as a transdermal drug delivery carrier. Pharm. Dev. Technol., 19, 164-172 (2014).

14) Ngawhirunpat $T$, Panomsuk S, Opanasopit P, Rojanarata T, Hatanaka T. Comparison of the percutaneous absorption of hydrophilic and lipophilic compounds in shed snake skin and human skin. Pharmazie, 61, 331-335 (2006).

15) Duangjit S, Opanasopit P, Rojanarata T, Ngawhirunpat T. Evaluation of meloxicam-loaded cationic transfersomes as transdermal drug delivery carriers. AAPS PharmSciTech, 14, 133-140 (2013).

16) Duangjit S, Pamornpathomkul B, Opanasopit P, Rojanarata $T$, Obata Y, Takayama K, Ngawhirunpat T. Role of the charge, carbon chain length, and content of surfactant on the skin penetration of meloxicam-loaded liposomes. Int. J. Nanomedicine, 9, 2005-2017 (2014).

17) Satish KM, Saugat A, Ameya AD. Application of simplex Lattice design in formulation and development of buoyant matrices of dipyridamole. J. App. Pharm. Sci., 2, 107-111 (2012).

18) Baroli B, López-Quintela MA, Delgado-Charro MB, Fadda AM, Blanco-Méndez J. Microemulsions for topical delivery of 8-methoxsalen. J. Control. Release, 69, 209-218 (2000).

19) Faust AC, Terpolilli R, Hughes DW. Management of an oral ingestion of transdermal fentanyl patches: a case report and literature review. Case Report Med., 2011, Article ID 495938, 4 pages, doi: 10.1155/2011/495938 (2011)

20) Rupenthal ID, Alany RG. Ocular drug celivery. Pharmaceutical manufacturing handbook: production and processes. (Gad SC ed.). John Wiley \& Sons, New Jersey, pp. 729-767 (2008).

21) Chrai SS, Robinson JR. Ocular evaluation of methylcellulose vehicle in albino rabbits. J. Pharm. Sci., 63, 1218-1223 (1974).

22) Mei Z, Chen H, Weng T, Yang Y, Yang X. Solid lipid nanoparticle and microemulsion for topical delivery of triptolide. Eur. J. Pharm. Biopharm., 56, 189-196 (2003).

23) Lee PJ, Langer R, Shastri VP. Novel microemulsion enhancer formulation for simultaneous transdermal delivery of hydrophilic and hydrophobic drugs. Pharm. Res., 20, 264-269 (2003).

24) Peltola S, Saarinen-Savolainen P, Kiesvaara J, Suhonen TM, Urtti A. Microemulsions for topical delivery of estradiol. Int. J. Pharm., 254, 99-107 (2003)

25) Behl CR, Flynn GL, Kurihara T, Harper N, Smith W, Higuchi WI, Ho NF, Pierson CL. Hydration and percutaneous absorption: I. Influence of hydration on alkanol permeation through hairless mouse skin. J. Invest. Dermatol., 75, 346-352 (1980).

26) Hathout RM, Woodman TJ, Mansour S, Mortada ND, Geneidi AS, Guy RH. Microemulsion formulations for the transdermal delivery of testosterone. Eur. J. Pharm. Sci., 40, 188-196 (2010).

27) Takahashi K, Tamagawa S, Katagi T, Yoshitomi H, Kamada A, Rytting JH, Nishihata T, Mizuno N. In vitro transport of sodium diclofenac across rat abdominal skin: effect of selection of oleaginous component and the addition of alcohols to the vehicle. Chem. Pharm. Bull., 39, 154-158 (1991).

28) Williams AC. Transdermal and topical drug delivery. Pharmaceutical Press, London (2003).

29) Ge S, Lin Y, Lu H, Li Q, He J, Chen B, Wu C, Xu Y. Percutaneous delivery of econazole using microemulsion as vehicle: Formulation, evaluation and vesicle-skin interaction. Int. J. Pharm., 465, 120-131 (2014)

30) Duangjit S, Obata Y, Sano H, Kikuchi S, Onuki Y, Opanasopit P, Ngawhirunpat T, Maitani Y, Takayama K. Menthosomes, novel ultradeformable vesicles for transdermal drug delivery: Optimization and characterization. Biol. Pharm. Bull., 35, 1720-1728 (2012).

31) Obata Y, Ashitaka Y, Kikuchi S, Isowa K, Takayama K. A statistical approach to the development of a transdermal delivery system for ondansetron. Int. J. Pharm., 399, 87-93 (2010).

32) Schmalfuß U, Neubert R, Wohlrab W. Modification of drug penetration into human skin using microemulsions. J. Control. Release, 46, 279-285 (1997).

33) Teichmann A, Heuschkel S, Jacobi U, Presse G, Neubert RHH, Sterry W, Lademann J. Comparison of stratum corneum penetration and localization of a lipophilic model drug applied in an o/w microemulsion and an amphiphilic cream. Eur. J. Pharm. Biopharm., 67, 699-706 (2007).

34) Garidel P, Fölting B, Schaller I, Kerth A. The microstructure of the stratum corneum lipid barrier: Mid-infrared spectroscopic studies of hydrated ceramide: palmitic acid:cholesterol model systems. Biophys. Chem., 150, 144-156 (2010).

35) Yu G, Stojadinovic O, Tomin-Canic M, Flach CR, Mendelsohn R. Infrared microscopic immaging of cutaneous would healing: lipid conformation in the migrating epithelial tongue. J. Biomed. Opt., 17, 0960091 (2012)

36) Mizushima H, Fukasawa J-I, Suzuki T. Phase behavior of artificial stratum corneum lipids containing a synthetic pseudo-ceramide: a study of the function of cholesterol. J. Lipid Res., 37, 361-367 (1996).

37) Ghanem A-H, Mahmoud H, Higuchi W, Rohr UD, Borsadia S, Liu P, Fox JL, Good WR. The effects of ethanol on the transport of $\beta$-estradiol and other permeants in hairless mouse skin. II. A new quantitative approach. J. Control. Release, 6, 75-83 (1987). 